\title{
A disabling combination: fatigue and depression ${ }^{\dagger}$
}

\author{
Stephanie S. Leone
}

\section{Summary}

Fatigue and common psychiatric symptoms - depression in particular - have consistently been found to be strongly associated. All have a negative impact on functional status, but evidence suggests that functional impairment is especially marked when they co-occur. Therefore, it is

pertinent to understand the relationship between fatigue and depression.

\section{Declaration of interest}

None.
Stephanie S. Leone (pictured) is a postdoctoral research fellow at the Department of General Practice and EMGO Institute of Health and Care Research at the VU University Medical Center, Amsterdam, The Netherlands.

Although fatigue has been the focus of a substantial amount of research, it generally remains an elusive concept to grasp. However, one of the most consistent findings in research on fatigue has been the strong relationship it has with common psychiatric symptoms, and depression in particular. ${ }^{1,2}$ The association between fatigue and depression holds across different healthcare settings and populations, from community samples to those in specialist care. ${ }^{3}$ In this issue of the Journal, Ball et al report on a population-based twin study conducted in Sri Lanka which aimed to assess the prevalence and aetiology of fatigue and its overlap with depression. Before considering their interesting results, I will touch upon some issues relating to fatigue and its relationship with depression.

\section{Co-occurrence and impairment}

An important reason to try to understand and disentangle the relationship between fatigue and depression concerns the functional impairment that is associated with their co-occurrence. Chronic fatigue and chronic fatigue syndrome (CFS) are associated with considerable functional impairment and disability be it in a social, physical or occupational sense. ${ }^{5,6}$ However, the co-occurrence of chronic fatigue or CFS with psychiatric symptoms such as depression is associated with greater functional impairment than when it occurs alone. The presence of psychiatric symptoms in individuals with chronic fatigue or CFS has also been found to be predictive of a poor outcome in terms of fatigue complaints as well as impairment. ${ }^{6}$

\section{Relationship between fatigue and depression}

Fatigue, in the same way as depression, can be defined in broad or narrow terms according to severity, duration and additional criteria (e.g. somatic symptoms, disability). The narrowest definitions being those for the syndromes or illnesses related to

'See pp. 106-113, this issue. these symptoms: CFS and major depressive disorder respectively. Different studies use different definitions and it is sometimes argued that the use of stricter definitions for fatigue will reduce the overlap between fatigue and depression. However, empirical evidence suggests that the association between fatigue and depression becomes larger when a narrower, more exclusive definition for fatigue is used as opposed to a broader definition. ${ }^{2}$

The overlap in occurrence and symptoms between chronic fatigue/CFS and depression has led some to suggest that they are merely a form of depression. Research on CFS suggests that these conditions can be distinguished from each other in several ways. Importantly, they do not always co-occur. Despite the overlap in some symptoms there are also differences. Typically, central features of depression such as issues of guilt and self-esteem do not fit the phenomenology of CFS. ${ }^{1}$ Moreover, some biological differences have been observed, most notably the (reversed) regulation of the hypothalamic-pituitary-adrenal (HPA) axis associated with these conditions: a down-regulation of the HPA axis has been noted in CFS, whereas an up-regulation of the HPA axis has been noted in depression. ${ }^{7}$ Twin studies have shown that fatigue seems to have genetic and environmental risk factors that are independent from psychiatric symptoms. ${ }^{8}$ Additionally, a recent cohort study found different and specific risk factors for fatigue with psychiatric comorbidity (e.g. family history of psychiatric disorder) and fatigue without psychiatric comorbidity (e.g. having excessive energy as a child). ${ }^{9}$

So what comes first: fatigue or depression? Unfortunately the results are equivocal. There is evidence to suggest that depression may predispose individuals to subsequent fatigue. ${ }^{10}$ On the other hand, fatigue and depression have been found to predict and influence each other in time. ${ }^{11,12}$ It has also been suggested that their association could partly be due to some common risk factors that give rise to both. ${ }^{12}$ For some, fatigue will come first; for others, depression will come first, but for most it will probably be unclear. As mood and fatigue seem to be so intertwined and the underlying mechanism remains relatively unknown, it will be difficult to identify what comes first until we understand how fatigue and mood are related to each other.

\section{Western or global phenomenon?}

Ball et al's population-based twin study on the aetiology of fatigue and its overlap with depression ${ }^{4}$ is especially relevant because of its setting: Sri Lanka. As most research on fatigue is done in Western countries, the question arises: is fatigue and its association with depression and disability merely an issue in high-income Western 
countries? Previous research on fatigue in non-Western settings suggests that this is not the case. For example, chronic fatigue and CFS have been found to be valid constructs across different countries and settings. ${ }^{13}$ A World Health Organization study conducted in 14 countries found that although people from high-income countries report more fatigue, people from lowincome countries tend to present fatigue as a complaint to their doctor more often. ${ }^{14}$ Moreover, a comparative study of CFS in Brazil and the UK showed that the prevalence of chronic fatigue and CFS and its associated disability was comparable between the two countries, but that causal attributions for fatigue differed. ${ }^{15}$

Essentially, Ball et al's Sri Lankan study suggests that fatigue is also a problem in this non-Western, low-income setting: the prevalence of abnormal fatigue was $25.3 \%$ and that of prolonged fatigue was $1 \% .{ }^{4}$ Unfortunately, interpreting the prevalence of prolonged fatigue is somewhat hampered by the fact that there were some ambiguities surrounding a questionnaire item relevant to the definition of prolonged fatigue (but not to abnormal fatigue). Although a thorough procedure was followed in translating the questionnaire, this limitation underlines the importance of cross-cultural validation of such instruments.

Patterns in the aetiology of fatigue and its relationship with depression and disability were quite similar to those found in Western settings. Fatigue was found to be associated with lifetime ever depression and this association was stronger when considering a narrower rather than a broader definition of fatigue. Perhaps most importantly, those reporting both abnormal fatigue and lifetime ever depression had more social impairment than those reporting either complaint separately. This finding is especially interesting as depression did not have to be present at the time of the study. Environmental and familial factors were found to contribute to the overlap between fatigue and lifetime ever depression, but environmental factors seemed to play a slightly more important role in this sample as compared with findings in Western samples.

This study provides us with new insights into the cross-cultural comparison of fatigue complaints and its association with depression and functional impairment. Building on these findings by examining specific, modifiable risk factors for fatigue and psychiatric comorbidity could prove to be valuable to practice. Moreover, it would also be interesting to learn more about whether specific risk factors differ between Western and non-Western countries.

\section{Conclusions}

Fatigue is a complex concept that continues to challenge researchers and practitioners alike. Its relationship with depression and functional impairment is a complex one, but an important one to untangle nonetheless. Moreover, Ball et al's study ${ }^{4}$ is an example of the emerging evidence that chronic fatigue and its association with functional impairment - especially when it co-occurs with depression - is not merely a 'Western' problem, but rather a global one.

In their review, Ross et al ask the question whether patients with CFS may be served better by focusing less on the medical mystery and more on the functional consequences. ${ }^{5}$ Preventing and treating the primary problem of chronic fatigue is naturally an important objective and it follows that unravelling the mystery of how fatigue develops and how it becomes associated with depression remains an important focus of research. However, because of the level of disability associated with chronic fatigue (with and without depression), focusing on the nature, identification and treatment of functional impairment at different stages in the course of fatigue could possibly help to reduce the impact that chronic fatigue has on the lives of those suffering from it.

Stephanie S. Leone, PhD, Department of General Practice and EMGO Institute for Health and Care Research, VU University Medical Center, Van der Boechorststraat 7, 1081 BT Amsterdam, The Netherlands. Email: s.leone@vumc.nl

First received 13 Jan 2010, final revision 3 May 2010, accepted 5 May 2010

\section{Acknowledgements}

Thanks to Professors H.E. van der Horst and S. Wessely for their useful comments and suggestions.

\section{References}

1 Afari N, Buchwald D. Chronic fatigue syndrome: a review. Am J Psychiatry 2003; 160: 221-36.

2 Skapinakis $P$, Lewis G, Mavreas V. Unexplained fatigue syndromes in a multinational primary care sample: specificity of definition and prevalence and distinctiveness from depression and generalized anxiety. Am J Psychiatry 2003; 160: 785-7.

3 Skapinakis P, Lewis G, Meltzer H. Clarifying the relationship between unexplained chronic fatigue and psychiatric morbidity: results from a community survey in Great Britain. Int Rev Psychiatry 2003; 15: 57-64.

4 Ball HA, Sumathipala A, Siribaddana SH, Kovas Y, Glozier N, McGuffin P et al. Aetiology of fatigue in Sri Lanka and its overlap with depression. Br J Psychiatry 2010; 197: 106-113.

5 Ross SD, Estok RP, Frame D, Stone LR, Ludensky V, Levine CB. Disability and chronic fatigue syndrome: a focus on function. Arch Intern Med 2004; 164: 1098-107.

6 Cairns R. Hotopf M. A systematic review describing the prognosis of chronic fatigue syndrome. Occup Med 2005; 55: 20-31.

7 Parker AJ, Wessely S, Cleare AJ. The neuroendocrinology of chronic fatigue syndrome and fibromyalgia. Psychol Med 2001; 31: 1331-45.

8 Hickie I, Kirk K, Martin N. Unique genetic and environmental determinants of prolonged fatigue: a twin study. Psychol Med 1999; 29: 259-68.

9 Harvey SB, Wessely S, Kuh D, Hotopf M. The relationship between fatigue and psychiatric disorders: evidence for the concept of neurasthenia. J Psychosom Res 2009; 66: 445-54.

10 Harvey SB, Wadsworth $M$, Wessely S, Hotopf $M$. The relationship between prior psychiatric disorder and chronic fatigue: evidence from a national birth cohort study. Psychol Med 2008; 38: 933-40.

11 Huibers MJH, Leone SS, van Amelsvoort LGPM, Kant I, Knottnerus JA. Associations of fatigue and depression among fatigued employees over time: a 4-year follow-up study. J Psychosom Res 2007; 63: 137-42.

12 Skapinakis $P$, Lewis $G$, Mavreas $V$. Temporal relations between unexplained fatigue and depression: longitudinal data from an international study in primary care. Psychosom Med 2004; 66: 330-5.

13 Hickie I, Davenport T, Vernon SD, Nisenbaum R, Reeves WC, Hadzi-Pavlovic D et al. Are chronic fatigue and chronic fatigue syndrome valid clinical entities across countries and health-care settings? Aust N Z J Psychiatry 2009; 43: 25-35.

14 Skapinakis $P$, Lewis G, Mavreas V. Cross-cultural differences in the epidemiology of unexplained fatigue syndromes in primary care. Br J Psychiatry 2003; 182: 205-9.

15 Cho HJ, Menezes PR, Hotopf M, Bhugra D, Wessely S. Comparative epidemiology of chronic fatigue syndrome in Brazilian and British primary care: prevalence and recognition. Br J Psychiatry 2009; 194: 117-22. 